OPEN ACCESS

Edited by:

Andre P. Vieira,

University of São Paulo, Brazil

Reviewed by:

José Ricardo G. Mendonça,

University of São Paulo, Brazil

Hernando Quevedo,

National Autonomous University of

Mexico, Mexico

${ }^{*}$ Correspondence:

Q. H. LiU

quanhuiliu@gmail.com

Specialty section:

This article was submitted to Mathematical and Statistical Physics,

a section of the journal

Frontiers in Physics

Received: 11 March 2021 Accepted: 04 June 2021

Published: 02 July 2021

Citation:

Yu J S, Zhou X, Chen J F, Du WK, Wang $X$ and Liu QH (2021) Local

Shape of the Vapor-Liquid Critical

Point on the Thermodynamic Surface

and the van der Waals Equation

of State.

Front. Phys. 9:679083

doi: 10.3389/fphy.2021.679083

\section{Local Shape of the Vapor-Liquid Critical Point on the Thermodynamic Surface and the van der Waals Equation of State}

\author{
J. S. Yu, X. Zhou, J. F. Chen, W. K. Du, X. Wang and Q. H. Liu* \\ School for Theoretical Physics, School of Physics and Electronics, Hunan University, Changsha, China
}

Differential geometry is a powerful tool to analyze the vapor-liquid critical point on the surface of the thermodynamic equation of state. The existence of usual condition of the critical point $(\partial p / \partial V)_{T}=0$ requires the isothermal process, but the universality of the critical point is its independence of whatever process is taken, and so we can assume $(\partial p / \partial T)_{V}=0$. The distinction between the critical point and other points on the surface leads us to further assume that the critical point is geometrically represented by zero Gaussian curvature. A slight extension of the van der Waals equation of state is to letting the two parameters $a$ and $b$ in it vary with temperature, which then satisfies both assumptions and reproduces its usual form when the temperature is approximately the critical one.

Keywords: critical point, van der Waals equation of state, Gaussian curvature, saddle point, response functions

\section{INTRODUCTION}

In thermal physics, a critical point is the end point of a phase equilibrium curve, the pressure-temperature curve that designates conditions under which a liquid phase and a vapor phase can coexist. The critical point $\left(T_{C}, V_{C}\right.$, and $\left.p_{C}\right)$ in the $p V$ diagrams is determined by

$$
\left(\frac{\partial p}{\partial V}\right)_{T}=0,\left(\frac{\partial^{2} p}{\partial V^{2}}\right)_{T}=0
$$

together with the thermodynamic equation of state (EoS), where symbols $(T, V$, and $p$ ) have their usual meaning as in ordinary textbooks [1-5]. The phase transition exhibits critical slowing down, universality, scaling, etc., which reflects a fact that the details of the system play an insignificant role $[6,7]$. How to characterize the essence of the critical point is always an attractive topic. We note two seemly independent developments/facts. One is that the critical slowing down is its pathindependence [8-12], which means that starting from any thermodynamic state in the vicinity of a critical point to approaching it, the system has inherently slow timescales whatever thermodynamic processes are chosen. The second is that a geometrical description of a local point on a curved surface is irrespective of either the parameters chosen to label the point on the surface or the paths selected to approach it. The strong resemblance of these two facts suggests that a geometrical description of the critical point is advantageous. Based on this observation, we propose that the critical point is geometrically represented by zero Gaussian curvature on the thermodynamic EoS surface, together with some physical assumptions. We hope to use this proposal to resolve a long-standing problem associated with the van der Waals (vdW) EoS. 
The most prominent aspect of the vdW EoS is that it captures many of the qualitative features of the liquid-vapor phase transition with the possible help of Maxwell's equal area rule. The vdW EoS was essentially presented by van der Waals in his $1873 \mathrm{PhD}$ thesis [13] (but explicitly given later [14]), and for this, he was awarded the Nobel Prize in Physics 1910 [13-17]. The $\mathrm{vdW}$ EoS is well-known as

$$
p=\frac{n R T}{V-n b}-\frac{n^{2} a}{V^{2}}
$$

where two parameters, $a$ and $b$, can be estimated from the critical point and considered constants, which are specific for each substance, and other symbols $(n, R)$ also have their usual meaning as in ordinary textbooks [1-5]. For one mole fluid, $n=1$, and the values of $T_{C}, V_{C}$, and $p_{C}$ are given in terms of $a$ and $b$ parameters [1-5] as

$$
T_{C}=\frac{8 a}{27 R b}, p_{C}=\frac{a}{27 b^{2}}, \text { and } V_{C}=3 b .
$$

With these values, the vdW EoS can be transformed into the following dimensionless form:

$$
p^{*}=\frac{8}{3} \frac{t^{*}}{v^{*}-1 / 3}-\frac{3}{v^{*}},
$$

where

$$
t^{*} \equiv \frac{T}{T_{C}}, v^{*} \equiv \frac{V}{V_{C}}, p^{*} \equiv \frac{p}{p_{C}} .
$$

Eq. 4 is referred to as the law of corresponding states which holds for all kinds of fluid substances, which was also originated with the work of van der Waals in about 1873 [13], when he used the critical temperature and critical pressure to characterize a fluid. However, whether and how the vdW parameters $a$ and $b$ depend on the temperature $T$, and even more on the volume $V$, has been a problem of long history. van der Waals himself was well-aware of it [14] and remarked in his Nobel Prize speech that "I have never been able to consider that the last word had been said about the equation of state and I have continually returned to it during other studies. As early as $1873 \mathrm{I}$ recognized the possibility that $\mathrm{a}$ and $\mathrm{b}$ might vary with temperature, and it is well-known that Clausius even assumed the value of a to be inversely proportional to the absolute temperature" [13]. In fact, more than one century passed since the discovery of the vdW EoS, we do not have strong experimental evidence nor a compelling theoretical argument to indicate how $a$ and $b$ parameters might depend on the temperature and/or volume. We have some theoretical results in statistical mechanics, revealing some temperature dependence of $a$ and $b$, for instance, in the hard-sphere model [1-5], but these results are frequently obtained for a dilute fluid far from the critical point, and more importantly, they rely heavily on the specific model without universality which is inherent to thermodynamics.

The present paper thus addresses two problems. One is why we assume $(\partial p / \partial T)_{V}=0$ that is complementary to the first formula of Eq. (1) and why we propose that the critical point is geometrically represented by zero Gaussian curvature on the thermodynamic EoS surface. Another is to use the above assumptions to discuss the long-standing problem within thermodynamics. This paper is organized as follows. In Section 2, we prove a theorem stating that the local shape of the vapor-liquid critical point on the thermodynamic surface can never be an elliptic point, and in order to completely characterize the local shape of the critical point, we need two more response functions which are assumed to vanish at the critical point, implied by the critical slowing down observed in either the realistic experiments or the computer simulation of the phase transition [8-12]. The vanishing response functions lead to the zero Gaussian curvature. In Section 3, the vdW EoS is slightly extended such that the parameters $a$ and $b$ vary with the temperature $T$, which is thus capable of giving zero Gaussian curvature at the critical point, while the usual form of the vdW EoS fails. In Section 4, a brief summary of the present study is given.

In the present paper, we concentrate on the (interior) Gaussian curvature that is sufficient to specify the local shape of the twodimensional thermodynamic EoS surface, but we will also give the (exterior) mean curvature as a contrasting quantity. In geometry, the curvature is usually referred to as the interior one.

\section{LOCAL SHAPE OF THE VAPOR-LIQUID CRITICAL POINT ON THE EQUATION OF STATE SURFACE AND A PROPOSAL}

In differential geometry, the local shapes of a two-dimensional curved surface are completely classified into three types: elliptic, hyperbolic, and parabolic, corresponding to the Gaussian curvature greater than, smaller than, or equal to zero, respectively [18-20]. For a thermodynamic EoS $p=$ $p(T, V)$ that can be treated as a two-dimensional surface in the three-dimensional flat space of coordinates $p, T$, and $V$, we now show that the vapor-liquid critical point cannot be an elliptic point.

In geometry, it is preferable to use the dimensionless equation of the EoS surface $p=p(T, V)$. The straightforward calculations can, respectively, give $H$ and Gaussian curvature $K$ :

$$
\begin{gathered}
H=\frac{\left(\frac{\partial^{2} p}{\partial V^{2}}\right)_{T}\left(\left(\frac{\partial p}{\partial T}\right)_{V}^{2}+1\right)+\left(\frac{\partial^{2} p}{\partial T^{2}}\right)_{V}\left(\left(\frac{\partial p}{\partial V}\right)_{T}^{2}+1\right)-2\left(\frac{\partial p}{\partial V}\right)_{T}\left(\frac{\partial p}{\partial T}\right)_{V}\left(\frac{\partial^{2} p}{\partial V \partial T}\right)}{2\left(\left(\frac{\partial p}{\partial V}\right)_{T}^{2}+\left(\frac{\partial p}{\partial T}\right)_{V}^{2}+1\right)^{3 / 2}}, \\
K=\frac{\left(\frac{\partial^{2} p}{\partial V^{2}}\right)_{T}\left(\frac{\partial^{2} p}{\partial T^{2}}\right)_{V}-\left(\frac{\partial^{2} p}{\partial V \partial T}\right)^{2}}{\left(\left(\frac{\partial p}{\partial V}\right)_{T}^{2}+\left(\frac{\partial p}{\partial T}\right)_{V}^{2}+1\right)^{2}}
\end{gathered}
$$

At the critical point the conditions in Eq. (1) apply, we have the mean curvature $H_{C}$ and Gaussian curvature $K_{C}$, respectively, as follows:

$$
H_{C}=\frac{\left(\frac{\partial^{2} p}{\partial T^{2}}\right)_{V}}{2\left(\left(\frac{\partial p}{\partial T}\right)_{V}^{2}+1\right)^{3 / 2}}, K_{C}=-\frac{\left(\frac{\partial^{2} p}{\partial V \partial T}\right)^{2}}{\left(\left(\frac{\partial p}{\partial T}\right)_{V}^{2}+1\right)^{2}},
$$


which shows that $K \leq 0$. Thus, we in fact prove a theorem that the local shape of the vapor-liquid critical point on the thermodynamic surface can never be an elliptic point.

To illustrate the mean and Gaussian curvature of the surface of the thermodynamic EoS, let us first consider two simple systems. For an incompressible liquid EoS: $V=$ const., which is a flat plane, both curvatures are zero. The ideal gas EoS surface, $p=n R T / V$, can be rewritten as a dimensionless one $p^{*} v^{*}=t^{*}$ with a reference point $\left(p_{0}, V_{0}, T_{0}\left(=p_{0} V_{0} /(n R)\right)\right)$, where $t^{*} \equiv T / T_{0}, \quad v^{*} \equiv V / V_{0}$, and $p^{*} \equiv p / p_{0}$. The mean curvature $H$ and Gaussian curvature $K$ are, respectively,

$$
H=\frac{t^{*} v^{* 3}}{\left(t^{* 2}+v^{* 2}+v^{* 4}\right)^{3 / 2}}, K=-\frac{v^{* 4}}{\left(t^{* 2}+v^{* 2}+v^{* 4}\right)^{2}} .
$$

Since the Gaussian curvature $K<0$ is negative definite, every point on the ideal gas EoS surface is saddle.

Now, we examine the vdW EoS surface [Eq. (2)], and it is preferable to use the dimensionless form [Eq. (4)]. The mean curvature $H$ and Gaussian curvature $K$ are, respectively,

$$
H=9 v^{* 5}\left(3 v^{*}-1\right)^{3} \frac{F_{1}\left(t^{*}, v^{*}\right)}{\left(F_{2}\left(t^{*}, v^{*}\right)\right)^{3 / 2}}, K=-\frac{576\left(3 v^{*}-1\right)^{4} v^{* 12}}{\left(F_{2}\left(t^{*}, v^{*}\right)\right)^{2}},
$$

where

$$
\begin{gathered}
F_{1}\left(t^{*}, v^{*}\right)=8 t^{*} v^{* 4}-27 v^{* 3}+27 v^{* 2}-73 v^{*}+65, \\
F_{2}\left(t^{*}, v^{*}\right)=576 t^{* 2} v^{*}-2592 t^{*} v^{* 5}+1728 t^{*} v^{* 4}-288 t^{*} v^{* 3} \\
+81 v^{* 10}-108 v^{* 9}+630 v^{* 8}-396 v^{* 7}+65 v^{* 6} \\
+2916 v^{* 4}-3888 v^{* 3}+1944 v^{* 2}-432 v^{*}+36 .
\end{gathered}
$$

At the critical point $\left(t^{*}, v^{*}\right)=(1,1)$, we have, respectively,

$$
H_{C}=0, K_{C}=-\frac{36}{289} \approx-0.125 \text {. }
$$

The negative Gaussian curvature $K_{C} \approx-0.125$ indicates that the critical point is a hyperbolic point, more precisely, a saddle point [20].

A comparison of the Gaussian curvatures in Eq. (9) for the ideal gas EoS and Eq. (13) for the vdW EoS suggests that there is no qualitative difference in between. It is a little bit odd, for we believe that a realistic EoS differs from the ideal gas EoS in the qualitative sense, rather than a quantitative one. By the critical point in the $P V$ diagram, we mean a stationary inflection point in the constant-temperature line, critical isotherm, whose location is determined by two equations in Eq. (1). However, if one approaches this point from an isobaric process or an isovolumetric process, or a more complicated process, we do not know whether such a point exhibits the same singularity. Therefore, we must seek for a general condition for the critical point, independently of thermodynamic paths.

At the local point of the thermodynamic EoS surface $p=p(T, V)$, the tangential plane is spanned by two independent vectors $\left(\partial_{T}, \partial_{V}\right)$. At the critical point $\left(T_{C}, V_{C}\right)$, we have $(\partial p / \partial V)_{T}=0$ in Eq. (1) which means the existence of a limit along the isotherm. Such a limit must exist along the isotherm ( $T=$ const. $)$ or along the isovolumetric line $(V=$ const. $)$, implying that we can further impose $(\partial p / \partial T)_{V}=0$ at the critical point.
Another condition is inspired by the Gaussian curvature that is independent of the detailed structure of matter, and the simplest assumption is $K_{C}=0$, implying $\partial^{2} p / \partial V \partial T=0$. In sum, we propose two additional conditions for the critical point on the EoS surface $p=p(T, V)$ :

$$
(\partial p / \partial T)_{V}=0, \partial^{2} p / \partial V \partial T=0 .
$$

It is worth mentioning that, in contrast to the realistic experiments which seem hard to measure these two response functions near the critical point, the computer simulations are more feasible [8-11], which shows that the critical slowing down is really an overall phenomenon no matter what path is chosen to approach the critical point.

\section{THE PROPOSAL AND TEMPERATURE DEPENDENCE OF VAN DER WAALS PARAMETERS $A$ AND $B$}

We are confident that the vdW EoS with the constant parameters $a$ and $b$ is not satisfactory for the following two senses. The first is that the Gaussian curvature at the critical point is $K_{C} \approx-0.125$ in Eq. (13) which is not qualitatively different from other points except the limiting situation. The second is that the value $K_{C} \approx-0.125$ manifestly depends on a special thermodynamic path, i.e., isotherm in Eq. (1). Fortunately, the vdW EoS can be adapted for removal of these weaknesses.

The slightest extension of the vdW EoS is to let the two constants $a$ and $b$ in the vdW EoS, Eq. (2), depend on the temperature as $a \rightarrow a(T)$ and $b \rightarrow b(T)$. The critical values of $T_{C}, V_{C}$, and $p_{C}$ are entirely determined by $a\left(T_{C}\right)$ and $b\left(T_{C}\right)$ :

$$
T_{C}=\frac{8 a\left(T_{C}\right)}{27 R b\left(T_{C}\right)}, p_{C}=\frac{a\left(T_{C}\right)}{27 b\left(T_{C}\right)^{2}}, V_{C}=3 b\left(T_{C}\right) .
$$

With introduction of the dimensionless $\alpha\left(t^{*}\right)$ and $\beta\left(t^{*}\right)$ instead of $a(T)$ and $b(T)$ in the following:

$$
\alpha\left(t^{*}\right) \equiv \frac{a(T)}{a\left(T_{C}\right)}=\frac{a\left(t^{*} T_{C}\right)}{a\left(T_{C}\right)}, \beta\left(t^{*}\right) \equiv \frac{b(T)}{b\left(T_{C}\right)}=\frac{b\left(t^{*} T_{C}\right)}{b\left(T_{C}\right)},
$$

the law of corresponding states does not hold true anymore except in the special case, $\alpha=$ const. and $\beta=$ const., and we have instead the dimensionless extended vdW EoS:

$$
p^{*}=\frac{8}{3} \frac{t^{*}}{v^{*}-\beta\left(t^{*}\right) / 3}-\frac{3 \alpha\left(t^{*}\right)}{v^{* 2}} \text {. }
$$

Near the critical point, we assume that $a(T)$ and $b(T)$ parameters take the following forms:

$$
\begin{aligned}
& a(T) \approx a\left(T_{C}\right)+a^{\prime}\left(T_{C}\right)\left(T-T_{C}\right)+\frac{1}{2} a^{\prime \prime}\left(T_{C}\right)^{2}\left(T-T_{C}\right)^{2}, \\
& b(T) \approx b\left(T_{C}\right)+b^{\prime}\left(T_{C}\right)\left(T-T_{C}\right)+\frac{1}{2} b^{\prime \prime}\left(T_{C}\right)^{2}\left(T-T_{C}\right)^{2},
\end{aligned}
$$

where

$$
g^{\prime}=\frac{d g}{d T}, g=a, b, a^{\prime}, b^{\prime}, \ldots
$$


The relations between set $\left(\alpha^{\prime}\left(T_{C}\right), \beta^{\prime}\left(T_{C}\right)\right)$ and set $\left(a^{\prime}\left(T_{C}\right), b^{\prime}\left(T_{C}\right)\right)$ are

$$
\alpha^{\prime}\left(t^{*}=1\right)=T_{C} \frac{a^{\prime}\left(T_{C}\right)}{a\left(T_{C}\right)}, \beta^{\prime}\left(t^{*}=1\right)=T_{C} \frac{b^{\prime}\left(T_{C}\right)}{b\left(T_{C}\right)},
$$

where $\alpha^{\prime}=d \alpha / d t^{*}$ and $\beta^{\prime}=d \beta / d t^{*}$.

The mean curvature $H$ and Gaussian curvature $K$ of the dimensionless extended vdW EoS surface have very long expressions of the complicated structure. However, the expressions for both $H$ and $K$ at the critical point $\left(t^{*}, v^{*}, \alpha, \beta\right)=$ $(1,1,1,1)$ are simply

$$
H_{C}=\frac{2 \beta^{\prime}\left(\beta^{\prime}+2\right)-3 \alpha^{\prime \prime}+2 \beta^{\prime \prime}}{2\left(G\left(t^{*}, v^{*}\right)\right)^{3 / 2}}, K_{C}=-\frac{36\left(1-\alpha^{\prime}+\beta^{\prime}\right)^{2}}{\left(G\left(t^{*}, v^{*}\right)\right)^{2}},
$$

where

$$
G\left(t^{*}, v^{*}\right)=4 \beta^{\prime^{2}}+9 \alpha^{\prime^{2}}-12 \alpha^{\prime} \beta^{\prime}+16 \beta^{\prime}-24 \alpha^{\prime}+17 .
$$

The distinctive feature of the extended $v d W E o S$ is that it contains two possible local shapes at the critical point: hyperbolic and parabolic, for $K_{C} \leq 0$. The case $K_{C}=0$ can be realized provided

$$
1-\alpha^{\prime}+\beta^{\prime}=0
$$

Note that two response functions $(\partial p / \partial T)_{V}$, and their partial derivative with respect to volume, $\left(\partial^{2} p / \partial V \partial T\right)$, produce values at the critical point $\left(T_{C}, V_{C}, p_{C}\right)$, respectively, as follows:

$$
\begin{gathered}
\left(\frac{\partial p}{\partial T}\right)_{V}=\frac{9 R T_{C} b^{\prime}\left(T_{C}\right)-4 a^{\prime}\left(T_{C}\right)+18 R b\left(T_{C}\right)}{36\left(b\left(T_{C}\right)\right)^{2}}, \\
\frac{\partial^{2} p}{\partial V \partial T}=-\frac{27 R T_{C} b^{\prime}\left(T_{C}\right)-8 a^{\prime}\left(T_{C}\right)+27 R b\left(T_{C}\right)}{108\left(b\left(T_{C}\right)\right)^{3}} .
\end{gathered}
$$

These two values are sufficient to completely fix two derivatives $\left(a^{\prime}\left(T_{C}\right), b^{\prime}\left(T_{C}\right)\right)$, given that the parameter $b\left(T_{C}\right)$ in Eqs. (24a,b) is given by the magnitude of the molar critical volume via Eq. (15).

Now let us examine situations where both response functions in Eq. (14) vanish at the critical point. First, once the second response function vanishes at the critical point, $\left(\partial^{2} p / \partial V \partial T\right)=0$, i.e., $27 R T_{C} b^{\prime}\left(T_{C}\right)-8 a^{\prime}\left(T_{C}\right)+27 R b\left(T_{C}\right)=0$ from Eq. (24b), we have from the relations in Eq. (20) that

$$
27 R b\left(T_{C}\right) \beta^{\prime}-8 \alpha^{\prime} a\left(T_{C}\right)+27 R T_{C} b\left(T_{C}\right)=0,
$$

which reproduces $1-\alpha^{\prime}+\beta^{\prime}=0$ in Eq. (23) with $27 T_{C} R b\left(T_{C}\right)=$ $8 a\left(T_{C}\right)$ in Eq. (15). Second, once the first response function in Eq. (14) vanishes at the critical point, $(\partial p / \partial T)_{V}=0$, i.e., $9 R T_{C} b^{\prime}\left(T_{C}\right)-4 a^{\prime}\left(T_{C}\right)+18 R b\left(T_{C}\right)=0$ from Eq. (24a), we have $4-3 \alpha^{\prime}+2 \beta^{\prime}=0$. An association of two equations $1-\alpha^{\prime}+$ $\beta^{\prime}=0$ and $4-3 \alpha^{\prime}+2 \beta^{\prime}=0$ yields

$$
\alpha^{\prime}=2, \beta^{\prime}=1 \text {.i.e., } a^{\prime}\left(T_{C}\right)=2 a\left(T_{C}\right) / T_{C}, b^{\prime}\left(T_{C}\right)=b\left(T_{C}\right) / T_{C} .
$$

With these values, we find that not only the critical point is locally flat but also $a(T)$ and $b(T)$ are accurate up to the first order of $\left(t^{*}-1\right)$ :

$$
\begin{gathered}
a(T) \approx a\left(T_{C}\right)+2 a\left(T_{C}\right)\left(t^{*}-1\right)=-a\left(T_{C}\right)+2 a\left(T_{C}\right) t^{*}, \\
b(T) \approx b\left(T_{C}\right)+b\left(T_{C}\right)\left(t^{*}-1\right)=b\left(T_{C}\right) t^{*} .
\end{gathered}
$$

When $t^{*} \approx 1$, i.e., $T \approx T_{C}, a(T) \approx a\left(T_{C}\right)$, and $b(T) \approx b\left(T_{C}\right)$, the usual form of vdW EoS is assumed. It is important to note that, from the two relations above, the usual vdW EoS is valid when the thermodynamic states are very close to the critical point, and $a(T)$ and $b(T)$ are also solely determined by $a\left(T_{C}\right)$ and $b\left(T_{C}\right)$.

\section{CONCLUSION}

Differential geometry is a powerful tool to reveal the intrinsic nature of the curved surface, and it is advantageous to analyze the critical point on the EoS surface. On the tangential plane of the critical point, the existence of limit $(\partial p / \partial V)_{T}=0$ requires the isothermal process. However, the essence of the critical point is its independence of whatever process is taken, and of detailed structure of matters. We can therefore assume $(\partial p / \partial V)_{v}=0$ and $K_{C}=0$ at the critical point on the EoS surface.

The vdW EoS is the simplest one to understand the liquid-gas transition. Since the vdW parameters $a$ and $b$ are constant, the Gaussian curvature is negative definite, and there is no distinction between the vdW EoS and the ideal gas EoS. According to our assumptions, the vdW EoS is slightly modified or extended such that the vdW parameters $a$ and $b$ vary with temperature, allowing for the presence of the zero Gaussian curvature at the critical point. Our approach sheds light on understanding the theoretical problem how the vdW parameters depend on the temperature.

\section{DATA AVAILABILITY STATEMENT}

The raw data supporting the conclusions of this article will be made available by the authors, without undue reservation.

\section{AUTHOR CONTRIBUTIONS}

All authors listed have made a substantial, direct, and intellectual contribution to the work and approved it for publication.

\section{FUNDING}

This work was financially supported by the National Natural Science Foundation of China under Grant No. 11675051. 


\section{REFERENCES}

1. Pathria RK, and Beale PD. Statistical Mechanics. 3rd ed. Oxford, UK: Butterworth-Heinemann (2011).

2. Huang K. Statistical Mechanics. 2nd ed. New York, NY: Wiley (1986).

3. Toda M, Kubo R, and Saito N. Statistical Physics I: Equilibrium Statistical Mechanics. 2nd ed. Berlin, Germany: Sringer-Verlag (2012).

4. Reif F. Fundamentals of Statistical and Thermal Physics. New York, NY: McGraw-Hill (1965).

5. Landau LD, and Lifshitz EM. Statistical Physics (I). 3rd ed. Oxford, UK: Pergamon (1980).

6. Kadanoff LP, Götze W, Hamblen D, Hecht R, Lewis EAS, and Palciauskas VV. Static Phenomena Near Critical Points: Theory and Experiment. Rev Mod Phys (1967) 39:395-431. doi:10.1103/revmodphys.39.395

7. Wilson KG. Renormalization Group and Critical Phenomena. I. Renormalization Group and the Kadanoff Scaling Picture. Phys Rev B (1971) 4:3174-83. doi:10.1103/physrevb.4.3174

8. Hohenberg PC, and Halperin BI. Theory of Dynamic Critical Phenomena. Rev Mod Phys (1977) 49:435-79. doi:10.1103/revmodphys.49.435

9. Wolff U. Critical Slowing Down. Nucl Phys B Proc Suppl (1990) 17:93-102. doi:10.1016/0920-5632(90)90224-i

10. Das M, and Green JR. Critical Fluctuations and Slowing Down of Chaos. Nat Commun (2019) 10:2155. doi:10.1038/s41467-019-10040-3

11. Brett T, Ajelli M, Liu Q-H, Krauland MG, Grefenstette JJ, and van Panhuis WG. Detecting Critical Slowing Down in High-Dimensional Epidemiological Systems. PLoS Comput Biol (2020) 16:e1007679. doi:10.1371/journal.pcbi.1007679

12. Kuehn C. A Mathematical Framework for Critical Transitions: Bifurcations, Fast-Slow Systems and Stochastic Dynamics. Physica D Nonlinear Phenomena (2011) 240:1020-35. doi:10.1016/j.physd.2011.02.012

13. van der Waals JD. On the Continuity Of the Gaseous And Liquid States. Leiden, Netherlands: Courier Corporation (1873).
14. van der Waals JD. The Equation of State for Gases and Liquids (December 12, 1910), Nobel Lectures Including Presentation Speeches and Laureates' Biographies. Physics. Amsterdam, Netherlands: Elsevier (1901). p. 254-1921.

15. Klein MJ. The Historical Origins of the Van Der Waals Equation. Physica (1974) 73:28-47. doi:10.1016/0031-8914(74)90224-9

16. Lebowitz JL, and Waisman EM. Statistical Mechanics of Simple Fluids: Beyond van Der Waals. Phys Today (1980) 33:24-30. doi:10.1063/1.2913994

17. Kontogeorgis GM, Privat R, and Jaubert J-N. Taking Another Look at the van der Waals Equation of State-Almost 150 Years Later. J Chem Eng Data (2019) 64:4619-37. doi:10.1021/acs.jced.9b00264

18. James Clerk Maxwell's Thermodynamic Surface, National Museum of Scotland. Available at: https://www.nms.ac.uk/explore-our-collections/ stories/science-and-technology/james-clerk-maxwell-inventions/james-clerkmaxwell/thermodynamic-surface/.

19. Kriz RD. Thermodynamic Case Study: Gibbs' Thermodynamic Graphical Method. Available at: https://esm.rkriz.net/classes/ESM4714/methods/Gibbs. html.

20. do Carmo MP. Differential Geometry of Curves and Surfaces. New York, NY: Prentice-Hall (1976). p. 146-7.

Conflict of Interest: The authors declare that the research was conducted in the absence of any commercial or financial relationships that could be construed as a potential conflict of interest.

Copyright (c) $2021 \mathrm{Yu}$, Zhou, Chen, Du, Wang and Liu. This is an open-access article distributed under the terms of the Creative Commons Attribution License (CC BY). The use, distribution or reproduction in other forums is permitted, provided the original author(s) and the copyright owner(s) are credited and that the original publication in this journal is cited, in accordance with accepted academic practice. No use, distribution or reproduction is permitted which does not comply with these terms. 